CORRECTION

https://doi.org/10.1038/s41586-019-1220-5

\title{
Publisher Correction: Resonant electro-optic frequency comb
}

Alfredo Rueda, Florian Sedlmeir, Madhuri Kumari,

Gerd Leuchs \& Harald G. L. Schwefel

Correction to: Nature https://doi.org/10.1038/s41586-019-1110-x, published online 17 April 2019.

In the Methods section of this Letter, owing to a typesetting error, the equations of motion using the Heisenberg picture were incorrectly shown as:

$$
\hat{\dot{a}}_{k}=\frac{i}{\hbar}\left[\hat{H}_{\mathrm{int}}, \hat{a}_{k}\right]=-i n_{\Omega} g\left(\hat{a}_{k-1}+\hat{a}_{k+1}\right)
$$

instead of as:

$$
\dot{\hat{a}}_{k}=\frac{i}{\hbar}\left[\hat{H}_{\mathrm{int}}, \hat{a}_{k}\right]=-i \sqrt{n_{\Omega}} g\left(\hat{a}_{k-1}+\hat{a}_{k+1}\right)
$$

This has been corrected online. 